CLINICAL REPORT

\author{
C.C. Quattrocchi \\ D. Longo \\ L.N. Delfino \\ M.R. Cilio \\ F. Piersigilli \\ M.D. Capua \\ G. Seganti \\ O. Danhaive \\ G. Fariello
}

\section{Dorsal Brain Stem Syndrome: MR Imaging Location of Brain Stem Tegmental Lesions in Neonates with Oral Motor Dysfunction}

\begin{abstract}
SUMMARY: The anatomic extent of brain stem damage may provide information about clinical outcome and prognosis in children with hypoxic-ischemic encephalopathy and oral motor dysfunction. The aim of this study was to retrospectively characterize the location and extent of brain stem lesions in children with oral motor dysfunction. From January 2005 to August 2009, 43 infants hospitalized at our institution were included in the study because of a history of hypoxic-ischemic events. Of this group, 14 patients showed oral motor dysfunction and brain stem tegmental lesions detected at MR imaging. MR imaging showed hypoxic-ischemic lesions in supra- and infratentorial areas. Six of 14 patients revealed only infratentorial lesions. Focal symmetric lesions of the tegmental brain stem were always present. The lesions appeared hyperintense on T2-weighted images and hypointense on IR images. We found a strong association $(P<.0001)$ between oral motor dysfunction and infratentorial lesions on MR imaging. Oral motor dysfunction was associated with brain stem tegmental lesions in posthypoxic-ischemic infants. The MR imaging examination should be directed to the brain stem, especially when a condition of prolonged gavage feeding is necessary in infants.
\end{abstract}

ABBREVIATIONS: APGAR = appearance, pulse, grimace, activity, respiration; $\mathrm{Cl}=$ confidence interval; IR = inversion recovery; SE = spin-echo
B rain MR imaging is the study of choice for assessing hypoxic-ischemic injury in neonates and infants. ${ }^{1,2}$ The pattern of brain injury suggests the pathogenetic mechanism and, together with the severity and extent of tissue damage, provides information about outcome and prognosis. Whereas conventional and advanced MR imaging protocols are appropriate for detecting supratentorial lesions, most routine MR imaging studies are not suitable for depicting brain stem hypoxic-ischemic lesions. Findings on advanced MR imaging techniques such as diffusion-weighted imaging and MR spectroscopy are difficult to interpret. In fact, to our knowledge, few reports on brain stem hypoxic-ischemic MR imaging findings in children have been reported in the literature.

At the brain stem level, involvement of cranial nerve nuclei and ascending-descending tracts has important chronic and potentially life-threatening clinical consequences.

Swallowing is a complex process with reflex activities that involve efferent and afferent fibers from the nuclei of cranial nerves V (cranial pons), VII, IX, X (caudal pons), and XII (medulla oblongata). The stereotypical sequence of oropharyngeal, laryngeal, and esophageal muscle contraction is elicited by stimulation of the pharynx by a food bolus and is derived from a central pattern generator. ${ }^{3}$ The generator neurons for the sequential motor response are located in the nucleus tract solitarius and in the dorsal medullary reticular formation.

Received December 31, 2009; accepted after revision February 16, 2010.

From the Departments of Radiology (C.C.Q., D.L., L.N.D., O.D., G.F.), Neuroscience (M.R.C., M.D.C.), and Medical Neonatology and Surgery (F.P., G.S., O.D.), IRCCS Ospedale Pediatrico Bambino Gesù, Rome, Italy.

C.C.O. and D.L. contributed equally to the concept and design of the study, the interpretation of results, and the preparation of the manuscript.

Please address correspondence to Carlo C. Quattrocchi, MD, PhD, Department of Radiology, IRCCS Ospedale Pediatrico Bambino Gesù, Piazza S. Onofrio, 4, 00100 Rome, Italy; e-mail: c.quattrocchi@unicampus.it

DOI 10.3174/ajnr.A2103
The tegmentum of the brain stem corresponds to the watershed area between the paramedian and circumferential branches of the basilar artery and is known to represent a vulnerable site to ischemic insults. ${ }^{4}$ Human neuropathologic studies have provided evidence of brain stem injury following hypoxic-ischemic events, showing bilateral symmetric necrotic areas in the tegmentum of the midbrain, pons, and medulla oblongata. ${ }^{5-8} \mathrm{MR}$ imaging has been reported to show pathologic findings such as symmetric high-signal-intensity lesions in the tegmentum of the upper medulla oblongata and caudal pons in neonates with dysphagia-gastroesophageal reflux complex. ${ }^{9}$

The aim of this study was to retrospectively characterize the MR imaging location and extent of brain stem lesions and to test their association with oral motor dysfunction in neonates and infants with pre-, peri-, and postnatal hypoxic-ischemic injury.

\section{Materials and Methods}

\section{Patients}

Patients with history of pre-, peri- or postnatal hypoxic-ischemic events who had undergone MR imaging examinations between January 2005 and August 2009 were included. Clinical and/or neurologic progression, metabolic diseases, central nervous system malformations, hydrocephalus, and infective and traumatic diseases were considered exclusion criteria. The 5-minute APGAR scores were collected. We considered patients with 5-minute APGAR scores of $>8$ as having no perinatal distress. Because our institution is a third-level hospital without an on-site maternity ward, consistent information about Sarnat scores, umbilical cord blood gases, or other neonatal encephalopathy scores were not available.

\section{Oral Motor Dysfunction}

At the acute phase, patients were evaluated for gastroesophageal reflux disease by esophageal $\mathrm{pH}$ or contrast upper gastrointestinal radi- 
ography and for the presence of arytenoid inflammation by fiberoptic laryngoscopy. Laryngeal penetration was defined by the presence of contrast medium beneath the epiglottis on a radiologic swallowing study, ${ }^{10}$ while tracheal aspiration was ascertained by the presence of radiology contrast medium or radionuclide in the trachea or distal airways on plain radiologic and cineradiologic or radionuclide studies. A senior neuroradiologist (G.F.) with expertise in abdominal conventional imaging read and interpreted the gastrointestinal studies. Percutaneous gastrostomy and placement of a jejunostomy tube or nasogastric tube were performed to allow gavage feeding.

\section{MR Imaging}

All images were obtained by using a 1.5T Magnetom Vision scanner (Siemens, Erlangen, Germany). Parental consent was obtained for each scan, and the study was based on guidelines approved by the hospital ethics committee. The infants were fed, swaddled, and placed in a vacuum fixation beanbag for immobilization. Nitrous oxide was administered for sedation by an anesthesiologist after parental consent. Sleeping infants were scanned with a quadrature knee coil under constant cardiorespiratory monitoring. The MR imaging protocol included coronal and axial SE T1-weighted sequences $(\mathrm{TR} / \mathrm{TE} /$ excitations $=400 / 15 \mathrm{~ms} / 2$ ), axial SE T2-weighted sequences $(\mathrm{TR} / \mathrm{TE} / \mathrm{excitations}=3800 / 81-120 \mathrm{~ms} / 1)$, and axial IR sequences $(\mathrm{TR} / \mathrm{TE} / \mathrm{TI}=8416 / 60 / 350 \mathrm{~ms})$, acquired with a $4-\mathrm{mm} \mathrm{sec}-$ tion thickness with no gap.

\section{Image and Data Analysis}

Brain conventional axial and coronal images were read to establish the location and extent of lesions at the supra- and infratentorial level. Images were evaluated on the MR imaging workstation, and conclusions were reached only after consensus of 3 neuroradiologists (C.C.Q., D.L., G.F.) and 1 fellow (L.N.D.). The presence, location, and extent of T2 hyperintense areas at the supra- or infratentorial levels were considered. Anagraphic, clinical, and MR imaging data were used to build a database on Excel (Microsoft, Bothell, Washington). Association studies were performed by applying the $\chi^{2}$ test on 2-way contingency tables, by using the tools provided at http:// statpages.org. Significance was determined at $P<.05$.

\section{Results}

\section{Patient Characteristics}

Forty-three patients (23 males, 20 females) with a history of pre-, peri-, and/or postnatal hypoxic-ischemic events were included in the study. Among them, 29 were born at term and 14 were preterm. History included intrauterine growth retardation, placenta abruption, umbilical cord around the neck at birth, cardiorespiratory depression, respiratory distress syndrome, severe apneas and bradycardia, and cardiac arrest with cardiopulmonary resuscitation. The mean gestational age was $37 \pm 4$ weeks. The mean 5 -minute APGAR score was $7 \pm 3$. Clinical presentation included hypotonia, spastic tetraplegia, seizures, psychomotor delay, vocal cord paralysis, apneas, respiratory insufficiency, and oral motor dysfunction in various combinations. All included patients showed a static clinical and neurologic condition up to a 6-month follow-up. Two patients died during the observation period: 1 after nosocomial pneumonia and the other from hypoxic-ischemic encephalopathy 7 days after asphyxia.

\section{MR Imaging}

The first MR imaging examination was performed at a mean age of $5.0 \pm 1.7$ months. In 8 patients, a follow-up MR imaging examination was performed at the mean age of $14 \pm 7.4$ months. In $37(86 \%)$ of the 43 patients included, focal or diffuse signal-intensity alterations were detected at MR imaging. Those alterations showed hyperintense signal on T2-weighted and hypointense signal on T1 and IR images. Among these, $54 \%(20 / 37)$ of patients showed only supratentorial lesions involving the basal ganglia, thalami, and/or subcortical/ periventricular white matter; the remaining $46 \%$ (17/37) showed infratentorial lesions, either alone (6/17) or associated with supratentorial findings (11/17). Imaging did not show pathologic findings in $6(14 \%)$ of the 43 patients included.

\section{Infratentorial Abnormalities}

Among brain stem levels, the medulla oblongata (6/6) and caudal pons (5/6) tegmenta were involved in the 6 patients with only infratentorial lesions (Figs 1 and 2). In this group of patients, we did not detect signal-intensity abnormalities at the midbrain level. In the remaining 11 patients with infraand supratentorial findings, the whole brain stem tegmentum was involved, including the midbrain (Fig 3).

The lesions appeared as focal and symmetric areas with hyperintense signal on T2-weighted images and hypointense signal on T1-weighted IR images, and they were located in the deep portion of the tegmentum, with sparing of the paramedian and most lateral regions. They appeared roughly circular on axial images with a columnar shape and variable extent on coronal images.

Eight patients underwent a follow-up study that showed persistence of T2-weighted hyperintensity, IR hypointensity as observed in lacunar infarctions, and a reduction of brain stem size. The cerebellum was spared by damage in all patients with only infratentorial lesions. In the group of patients with infraand supratentorial lesions, 54\% (6/11) showed cerebellar hypoplasia (vermis in 3/6 and bilateral hemispheres in 3/6) and $9 \%(1 / 11)$ showed cerebellar diffuse hyperintensity.

\section{Oral Motor Dysfunction and MR Imaging}

Suction-deglutition alterations and aspiration episodes were confirmed by radiologic and radionuclide findings with diagnosis of oral motor dysfunction in $42 \%$ (18/43) of the included patients. Among these, 13 patients underwent percutaneous gastrostomy or placement of a jejunostomy tube and 5 patients were fed through a nasogastric tube. In this group, MR imaging showed infratentorial lesions in $77.8 \%(14 / 18)$ of patients. Among these, 4 patients had a 5-minute APGAR score of $>8$. Of the 43 patients, 4 infants showed oral motor dysfunction in the absence of infratentorial lesions and 3 infants showed infratentorial findings in the absence of oral motor dysfunction. We found association $(P<.0001)$ between oral motor dysfunction and MR imaging infratentorial lesions with a calculated odds ratio of 25.7 (95\% CI, 5.21-125.8) and a relative risk of 5.35 (95\% CI, 2.46-10.48).

\section{Discussion}

Our study demonstrates involvement of the brain stem tegmentum in neonates and infants with hypoxic-ischemic encephalopathy. Damage to the brain stem is rarely reported in 

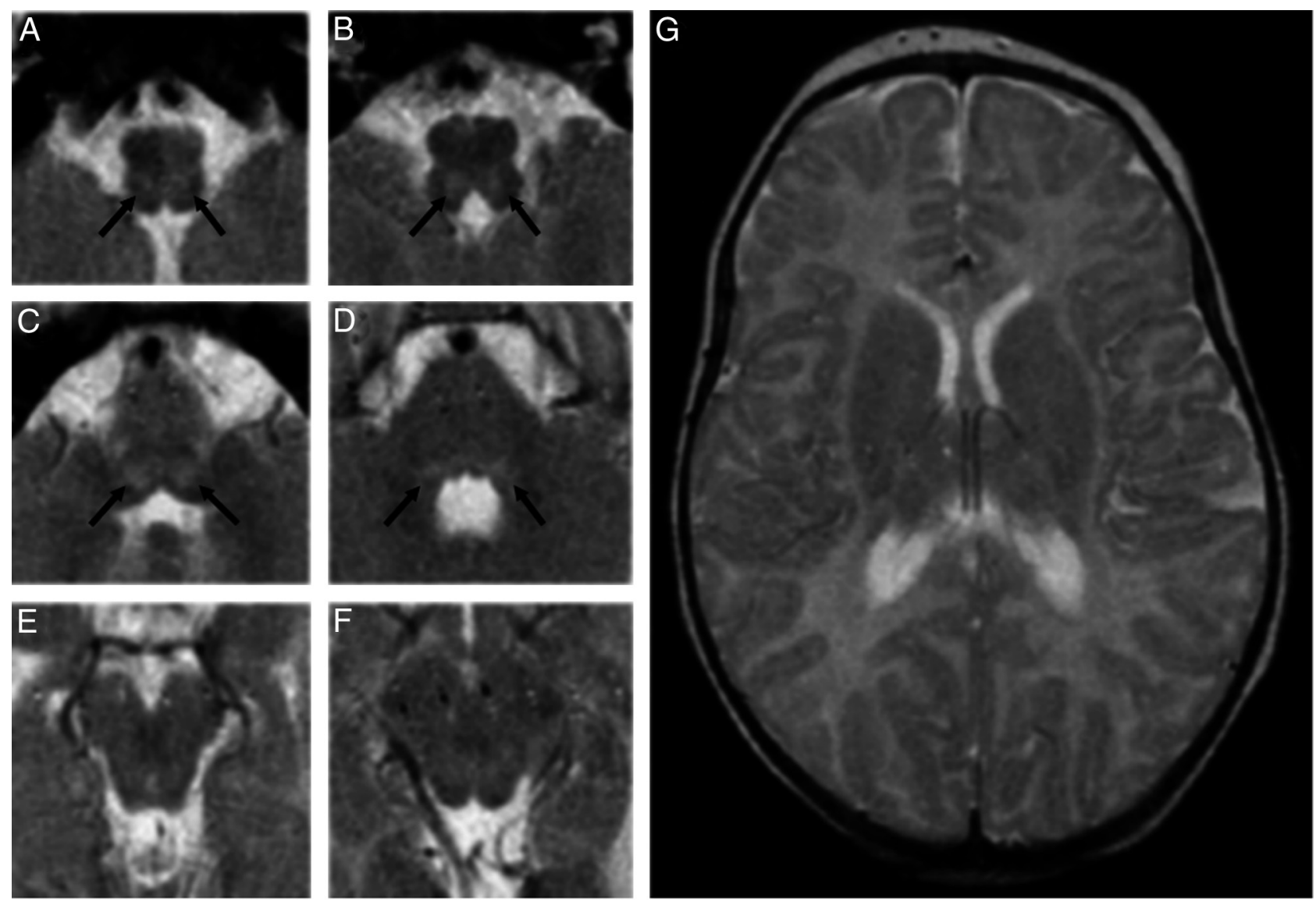

Fig 1. $A-D$, MR images show T2 focal, bilateral, and symmetric hyperintense lesions in the tegmentum at the level of the medulla oblongata (arrows in $A$ and $B$ ) and of caudal pons (arrows in $C$ ), whereas faint dorsal bilateral hyperintensity is seen at the level of the cranial pons (arrows in $D$ ). $E-G$, No signal-intensity or structural alterations are detected at the level of the midbrain $(E$ and $F)$ and the supratentorial structures $(G)$.

children, and only accurately performed MR imaging scans depict alterations of the brain stem. Focal bilateral signalintensity abnormalities have been localized in the tegmentum in a subset of term and near-term infants with or without a history of asphyxia. ${ }^{11,12}$ In prospective studies of neonates with hypoxic-ischemic encephalopathy, brain stem lesions are only sporadically mentioned. ${ }^{13-17}$

However, neuropathologic studies provide evidence of bilateral symmetric necrotic areas in the tegmentum of the midbrain, pons, and medulla oblongata in neonates with hypoxic-ischemic encephalopathy, including large parts of the medial and lateral reticular formation and numerous adjacent tegmental nuclei. ${ }^{4}$ Similar lesions were reported with a $90 \%$ prevalence in another series, associated with multifocal neuronal loss in the cortex and basal ganglia, ${ }^{5}$ in a 36-week-old infant with acute fetal distress ${ }^{6}$ and a 25 -week-old infant with prolonged bradycardia. $^{7}$

Brain stem alterations in neonates and infants are usually under-reported, probably due to the intrinsic limitations of MR imaging studies, such as the small size of the brain stem in infants and the fact that MR imaging protocols do not address the study of infratentorial structures in such unstable patients. Moreover, movement artifacts require sedation in neonates.

A group of patients with oral motor dysfunction, leading to protracted gavage feeding, showed brain stem MR imaging lesions. Multiple levels of the nervous system are involved in the control of swallowing. ${ }^{18}$ The primary afferents include cranial nerves V, VII, IX, X, and XII. Mastication, respiration, and swallowing are under the control of specific regions of the brain stem defined as pattern generators and are modulated by higher suprabulbar regions and sensory feedback. Oral function and swallowing involve complex pathways, located throughout the whole brain stem as well as several supratentorial structures in the cortex and basal ganglia. ${ }^{19}$ The brain stem tegmentum is known to be a vulnerable site, especially after hypoxic-ischemic events and asphyxia. A combination of hemodynamic and metabolic factors accounts for this brain stem selective vulnerability to hypoxia in the fetal and neonatal period. In fact, the dorsal brain stem has higher metabolic demands than the ventral brain stem, so that it could be selectively damaged under hypoxic-ischemic conditions. ${ }^{20}$

With regard to the vascularization, the brain stem, at the level of medulla oblongata and pons, is perfused by paramedian penetrating and short and long circumferential arteries branching from the basilar artery. ${ }^{3}$ This distribution determines a relatively avascular zone in the paramedian area of the brain stem during fetal life, and a watershed area in the tegmentum persists with time between the terminal perfusion zones of these arteries. ${ }^{20}$ This area includes cranial nerve IVXII nuclei, the solitary tract nucleus and ambiguous nucleus, the reticular formation, and ascending-descending tracts. Thus the lesions that we detected, as previously mentioned, may be directly related to the oral motor dysfunction. Detected injury was always bilateral and symmetric, with involve- 

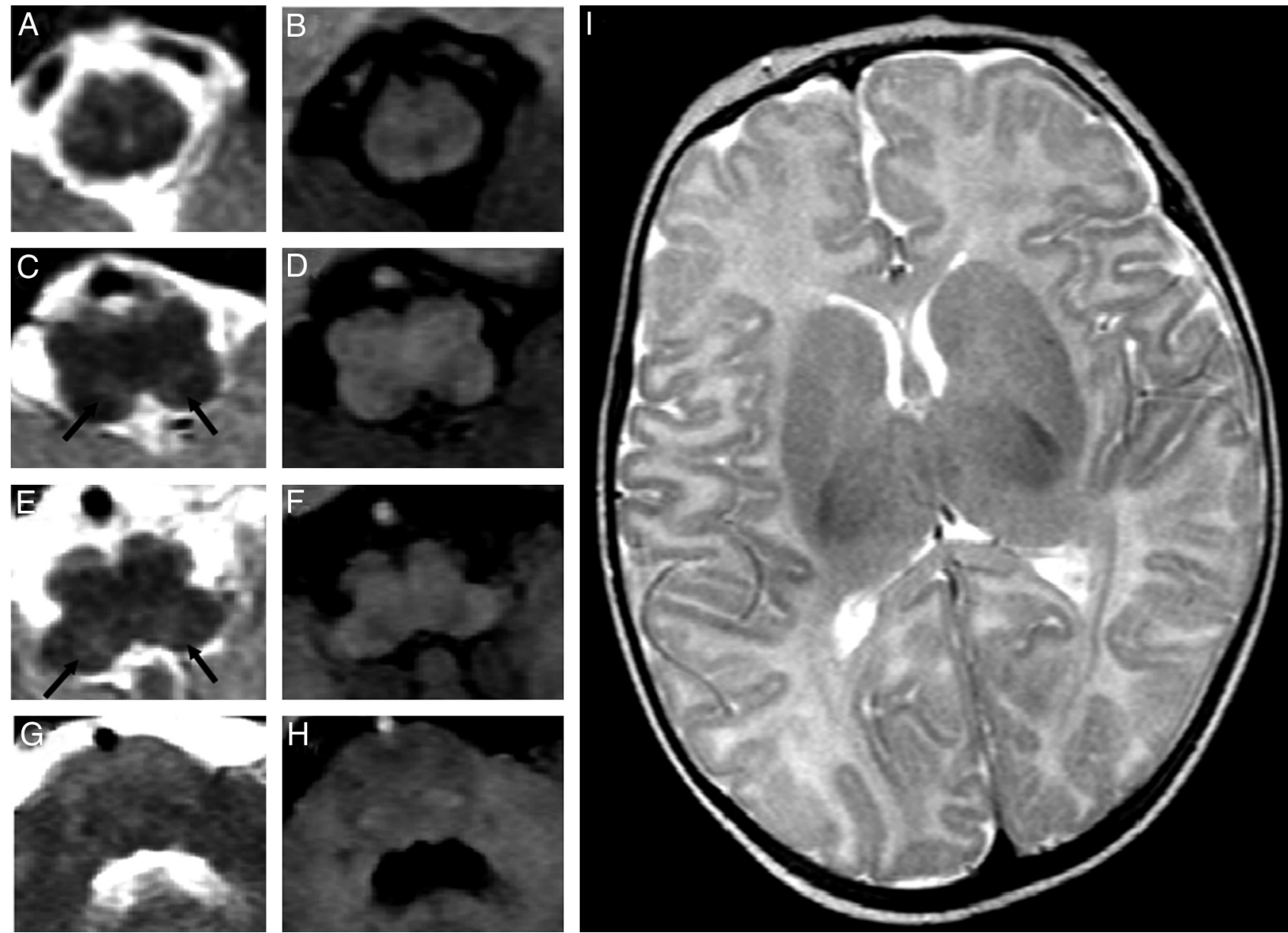

Fig 2. MR images demonstrate the presence of tegmental lesions appearing on T2-weighted $(A, C, E, G)$ and T1-weighted $(B, D, F, H)$ images. Focal, bilateral, and symmetric lesions (arrows in $C$ and $E$ ) show hyperintense signal intensity on T2-weighted images and faint hypointense signal intensity on T1-weighted weighted images. No signal-intensity or structural alterations are detected at the level of the cranial pons $(G$ and $H)$ and the supratentorial structures (/).
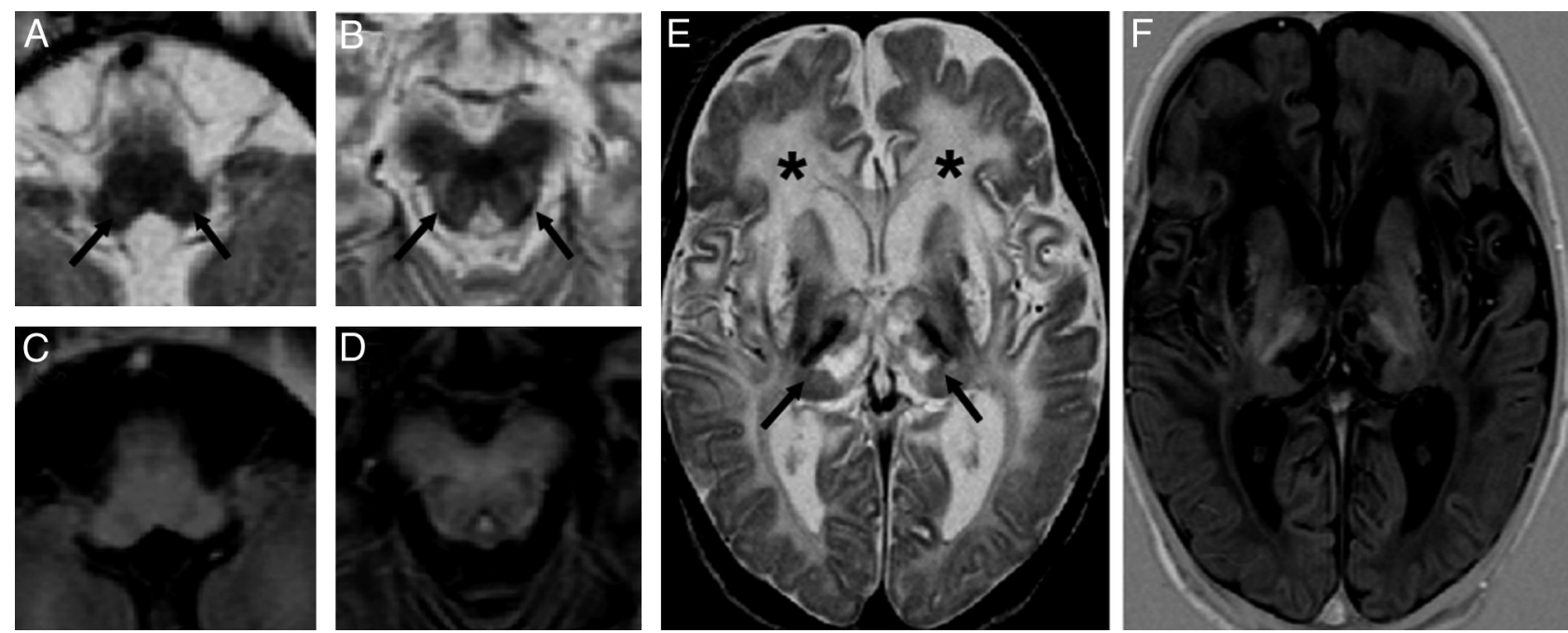

Fig 3. MR images show focal, bilateral, and symmetric T2 hyperintense lesions of the tegmentum (arrows in $A$ and $B$ ) at the level of the whole brain stem, including the medulla oblongata $(A$ and $C)$ and midbrain $(B$ and $D)$. Diffuse signal-intensity alterations are detected at the level of the subcortical and periventricular white matter (asterisks in $E$ ) and the subcortical nucle (arrows in $E$ ), as seen in hypoxic-ischemic encephalopathy. Lesions show hyperintense signal intensity on T2-weighted images $(A, B$, and $E$ ) and hypointense signal intensity on IR images $(C, D$, and $F)$.

ment of cranial nerves and reticular formation, sometimes including the respiratory center.

Among our patients, we could distinguish 2 groups: those with caudal pons and medulla oblongata lesions and those with whole brain stem lesions, including the midbrain with associated supratentorial lesions. On the basis of this consideration, we speculate that what has been previously defined as "dorsal brain stem syndrome" ${ }^{21}$ could be differentiated into 
a partial form involving the caudal pons and medulla and a complete form involving the whole brain stem and supratentorial structures. When injury involves a hypoxic-ischemic pathogenesis, selective vulnerability related to vascularization should be considered; the caudal pons and medulla oblongata, in fact, are only supplied by branches of the basilar artery with no mechanisms of flow compensation during hypotension. The midbrain, on the other hand, could be less vulnerable than lower brain stem levels, due to blood supply from perforating branches of the posterior cerebral arteries or superior cerebellar arteries. We are aware that this distinction appears to conflict with what has been previously demonstrated in primate models of fetal and neonatal hypoxia-ischemia by Myers. ${ }^{22}$ That study, in fact, showed that only severe and prolonged periods of hypoxia resulted in subcortical and brain stem infarctions, resembling the watershed tegmentum lesions in neonates.

On the other hand, we observed patients with only infratentorial brain stem lesions. Even though the small number of patients does not allow conclusive findings, the condition of polyidramnios or oligoidramnios in the group of patients with only infratentorial lesions may be considered a susceptibility factor that may increase the risk of tegmental lesions, even after short periods of hypoxia. ${ }^{20}$ Nevertheless, it is very difficult to compare results of complex clinical events with experimental conditions.

The retrospective design and the lack of information on some perinatal clinical conditions such as the Sarnat score and the umbilical cord blood gases, which allow one to somehow quantify the severity of hypoxic-ischemic damage, are the main limitations of this study. Moreover, these results should be confirmed and supported by analysis of larger samples of patients.

\section{Conclusions}

Brain stem lesions may be detected at MR imaging in posthypoxic-ischemic infants. They seem to occur consistently in tegmental areas consequent to both selective vulnerability and hypoperfusion. Oral motor dysfunction is associated with the presence of tegmental lesions, with or without supratentorial MR imaging findings. When gavage feeding is necessary in infants, the MR imaging examination should be directed toward the brain stem. This examination may be crucial in de- fining the clinical diagnosis of dorsal brain stem syndrome and determining the extent of the tegmental lesions.

\section{References}

1. Ment LR, Bada HS, Barnes P, et al. Practice parameter: neuroimaging of the neonate-report of the Quality Standards Subcommittee of the American Academy of Neurology and the Practice Committee of the Child Neurology Society. Neurology 2002;58:1726-38

2. Counsell SJ, Tranter SL, Rutherford MA. Magnetic resonance imaging of brain injury in the high-risk term infant. Semin Perinatol 2010;34:67-78

3. Jean A. Brain stem control of swallowing: neuronal network and cellular mechanisms. Physiol Rev 2001;81:929-69

4. Sarnat HB. Watershed infarcts in the fetal and neonatal brainstem: an aetiology of central hypoventilation, dysphagia, Möebius syndrome and micrognathia. Eur J Paediatr Neurol 2004;8:71-87

5. Schneider H, Ballowitz L, Schachinger H, et al. Anoxic encephalopathy with predominant involvement of basal ganglia, brain stem and spinal cord in the perinatal period: report on seven newborns. Acta Neuropathol 1975;32:287-98

6. Leech RW, Alvord EC Jr. Anoxic-ischemic encephalopathy in the human neonatal period: the significance of brain stem involvement. Arch Neurol 1977; 34:109-13

7. Natsume J, Watanabe K, Kuno K, et al. Clinical, neurophysiologic, and neuropathological features of an infant with brain damage of total asphyxia type (Myers). Pediatr Neurol 1995;13:61-64

8. Pindur J, Capin DM, Johnson MI, et al. Cystic brain stem necrosis in a premature infant after prolonged bradycardia. Acta Neuropathol 1992;83:667-69

9. Saito Y, Kawashima Y, Kondo A, et al. Dysphagia-gastroesophageal reflux complex: complications due to dysfunction of solitary tract nucleus-mediated vago-vagal reflex. Neuropediatrics 2006;37:115-20

10. Friedman B, Frazier JB. Deep laryngeal penetration as a predictor of aspiration. Dysphagia 2000;15:153-58

11. Barkovich AJ, Sargent SK. Profound asphyxia in the premature infant: imaging findings. AJNR Am J Neuroradiol 1995;16:1837-46

12. Sugama S, Eto Y. Brainstem lesions in children with perinatal brain injury. Pediatr Neurol 2003;28:212-15

13. Cowan F, Rutherford M, Groenendaal F, et al. Origin and timing of brain lesions in term infants with neonatal encephalopathy. Lancet 2003;361: $736-42$

14. Keeney SE, Adcock EW, McArdle CB. Prospective observations of 100 highrisk neonates by high-field ( 1.5 Tesla) magnetic resonance imaging of the central nervous system. II. Lesions associated with hypoxic-ischemic encephalopathy. Pediatrics 1991;87:431-38

15. Steinlin M, Dirr R, Martin E, et al. MRI following severe perinatal asphyxia: preliminary experience. Pediatr Neurol 1991;7:164-70

16. Miller SP, Ramaswamy V, Michelson D, et al. Patterns of brain injury in term neonatal encephalopathy. J Pediatr 2005;146:453-60

17. Baenziger O, Martin E, Steinlin M, et al. Early pattern recognition in severe perinatal asphyxia: a prospective MRI study. Neuroradiology 1993;35:437-42

18. Miller AJ. Deglutition. Physiol Rev 1982;62:129-84

19. Smith LH, DeMyer WE. Anatomy of the brainstem. Semin Pediatr Neurol 2003; 10:235-40

20. Leong S, Ashwell KW. Is there a zone of vascular vulnerability in the fetal brain stem? Neurotoxicol Teratol 1997;19:265-75

21. Saito Y. Reflections on the brainstem dysfunction in neurologically disabled children. Brain Dev 2009;31:529-36

22. Myers RE. Two patterns of perinatal brain damage and their conditions of occurrence. Am J Obstet Gynecol 1972;112:246-76 